\title{
EFAVIRENZ IN PREGNANCY
}

\author{
Polly Clayden ${ }^{1}$ \\ Vivian Black $^{2}$ \\ Andrew Boulle ${ }^{3}$ \\ Ashraf Hassen Coovadia ${ }^{4}$ \\ Francois Venter $^{2}$ \\ ${ }^{1}$ HIV i-Base, UK \\ ${ }^{2}$ Reproductive Health and HIV Research Unit, University of the Witwatersrand, Johannesburg \\ ${ }^{3}$ School of Public Health and Family Medicine, University of Cape Town \\ ${ }^{4}$ Head of HIV Services, Rahima Moosa Mother and Child Hospital and Department of Paediatrics and Child Health, University of the \\ Witwatersrand, Johannesburg
}

Clinical guidelines from the National Department of Health (DoH), South Africa, for prevention of mother-tochild transmission (PMTCT), revised in 2010, recommend that HIV-positive pregnant women with a CD4 count of 350 cells/ $\mu$ l or less commence lifelong antiretroviral therapy (ART). ${ }^{1}$

DoH guidance for women initiating ART in pregnancy in the public sector - on which the overwhelming majority of HIV-positive South Africans rely for their care - recommends they receive nevirapine with tenofovir and lamivudine or emtricitabine at any stage of gestation. In cases where a woman is already receiving ART with an efavirenz-based regimen, it is recommended that this should be substituted for nevirapine if she is still in the first trimester of pregnancy. Efavirenz is therefore contraindicated in pregnant women at any time during pregnancy; for those already receiving the drug, it is only switched in the first trimester.

The concern about the use of efavirenz in pregnancy dates back to preclinical studies. It is the only antiretroviral with preclinical primate data and in turn has the strongest US Food and Drug Administration (FDA) category and the most scrutiny during pregnancy. ${ }^{2}$ The drug also has the most conflicting recommendations, both from guidelines and product labelling.

This article is a summary of what we know (and do not know) about using efavirenz in pregnancy. We argue that reconsideration of the risk and benefits of this evidence, which has informed South African guidance, is warranted.

\section{MIXED MESSAGES FROM PRECLINICAL DATA, GUIDELINES AND LABELLING}

During the development of efavirenz, animal studies were conducted to assess its potential for birth defects. These primate data are likely to have made providers and regulators more aware of the potential risks with efavirenz.

Malformations were observed in three out of 20 monkey offspring from efavirenz-treated cynomolgus monkeys (versus none among 20 controls) in a developmental toxicity study. The monkeys were dosed throughout pregnancy with a dose resulting in plasma drug concentrations similar to those in humans given $600 \mathrm{mg} /$ day of efavirenz. Anencephaly and unilateral anophthalmia were observed in one monkey infant, micro-ophthalmia was observed in another, and cleft palate was seen in a third. As a consequence of this trial, efavirenz was classified as FDA category $C$.

In 2005, efavirenz was reclassified as FDA category $D$, indicating an established risk to the human fetus. This classification was based on three reports of myelomeningocele and one of Dandy-Walker syndrome to the Antiretroviral Pregnancy Register (APR). ${ }^{3}$ Each defect was reported retrospectively and therefore the relative risk cannot be calculated, as the denominator is unknown. Two of the reported cases of myelomeningocele (spina bifida) and the case of Dandy-Walker syndrome were reported in aborted fetuses.

The low quality of evidence regarding the safety of efavirenz in pregnancy has led to much uncertainty 
when making recommendations. This is particularly problematic for guidance in low- and middle-income countries such as South Africa, with few antiretroviral options and a public health approach. However, the APR provides some reassurance, as does a meta-analysis performed earlier this year. ${ }^{4}$

Both the registry and the meta-analysis suggest that a twofold increase in overall birth defects can be excluded, but some caution about the potential for increased neural tube defects remains because of the primate data. This, however, needs to be taken in the context of a low incidence of neural tube defects in the general population, which varies from country to country, and is in the range of $0.1 \%$ (South Africa is $0.36 \%$ ). With current data it is only possible to exclude a potential tenfold or higher increase in risk, but as the authors of the meta-analysis described below suggest, even a fivefold increase would give an overall increase of less than 1\%. It is also important to take into consideration that the neural tube closes by approximately 28 days' gestation, so the potential risk is for women receiving the drug in the first trimester. Since pregnancies are seldom recognised by this stage, if there is a risk it would be in women who conceive while already taking efavirenz.

This uncertainty has led to differences in interpretation when making recommendations. The World Health Organization (WHO) pregnancy guidelines recommend that efavirenz should not be initiated in the first trimester, but may be initiated in the second and third trimesters. ${ }^{5}$ The WHO adult guideline panel was unable to conclude from the evidence available whether there were benefits associated with the use of efavirenz compared with nevirapine in pregnant women after the first trimester and with higher or unknown CD4 cell counts. However, they note that more than half the panel members preferred efavirenz in these situations. ${ }^{6}$

US guidelines also state that efavirenz use can be considered after the first trimester. Other countries such as Zambia concur, although most European guidance is similar to South Africa. ${ }^{7-9}$

The British HIV Association (BHIVA) recommends: 'Until more robust data are available it remains advisable to avoid efavirenz for women who may conceive."10 These guidelines highlight two issues to consider for women who do conceive on efavirenz: the gestational age at presentation and the plasma half-life of efavirenz. They explain that, after stopping, it can take up to 3 weeks for efavirenz to clear from the plasma. Whether there is a key period during the first 6 weeks of fetal development when efavirenz affects central nervous system (CNS) development is unknown, as is the minimal teratogenic dose. They suggest 'discontinuing efavirenz after neural tube closure will not influence the outcome'.
Furthermore, the prescribing information accompanying the drug is inconsistent. Bristol-Meyers Squibb (BMS) markets the originator efavirenz product (sold as Sustiva in the USA, Canada and some countries in the European Union). Their package insert states: 'Fetal harm can occur when administered to a pregnant woman during the first trimester.'11

Merck Sharp and Dohme (MSD, who market efavirenz as Stocrin in South Africa and many other parts of the world) provides prescriber information for efavirenz that cautions against conception in patients already on efavirenz as opposed to the prescription of efavirenz in women already known to be pregnant, in line with the WHO and BHIVA recommendations focusing on very early gestational risk. Their package insert states that 'pregnancy should be avoided in women receiving Stocrin ${ }^{\circledR !}$ ! 12

The generic manufacturer Aspen states: 'The use of Aspen efavirenz during pregnancy is not recommended, as teratogenicity has been noted.' ${ }^{13}$

\section{THE ANTIRETROVIRAL PREGNANCY REGISTRY}

The APR is an international registry started in 1989 to prospectively monitor potential birth defects in infants exposed to antiretrovirals in utero. It is one of the largest ongoing pregnancy registries in the world.

The objectives of the registry are to provide early warning of major teratogenicity, estimate the risk of birth defects, and collect supplementary data from animal, clinical and epidemiological studies. Data collection is through voluntary enrolment by those providing health care to pregnant women exposed to antiretrovirals, and in turn infant follow-up. The registry has summaries of relevant data for all registered antiretrovirals, and reports are updated twice a year.

The majority of reports (84.9\%) are from the USA, with small numbers from elsewhere (e.g. 1.9\% from South Africa). One of the current goals of the registry is to increase non-US reporting.

As of the last review, through January 2010, the prevalence of birth defects per 100 live births among women with first-trimester exposure to any antiretroviral was 2.8\% (95\% confidence interval (CI) 2.3 - 3.3). At this review there were 13575 pregnancies enrolled in the registry, of which 11867 (87.4\%) reports, of which 5582 were first-trimester exposures, were used in the analysis. The overall rate reported is not significantly different from the prevalence of defects among women with initial exposure during the second and/or third trimester (2.5 per 100 live births). 
Only 14 defects have been recorded in 546 infants born following first-trimester efavirenz exposure (2.6\%, $95 \% \mathrm{Cl} 1.4-4.3 \%$ ). These include a single case of myelomeningocele and a single case of anophthalmia with severe oblique facial clefts and amniotic banding.

It is worth noting that since the incidence of myelomeningocele in the USA is 4.3 per 10000 live births it would be expected that the number of cases observed in the prospectively reported register should be greater than this overall.

\section{META-ANALYSIS}

Earlier this year, Ford et al. performed a systematic review of databases (to 2 February 2010) in order to identify observational cohorts reporting birth outcomes among infants exposed to maternal efavirenz during the first trimester of pregnancy. The findings from this analysis were published in AIDS in June 2010. Slightly modified data (to take in the updated APR report in January) were presented at IAS 2010. ${ }^{14}$ Besides birth defects of any kind the investigators looked at spontaneous abortions, termination of pregnancy, stillbirths and preterm delivery.

The investigators found that 16 studies met the inclusion criteria for the analysis. These included 11 prospective and 5 retrospective cohorts. Nine studies were conducted in low and middle-income countries. Six were European and one primarily in the USA. Eight were reported in journal articles, and 6 as conference abstracts, one (MTCT-Plus) was an unpublished cohort and one the APR report.

This analysis found a pooled non-significant relative risk for efavirenz versus non-efavirenz of 0.85 (95\% $\mathrm{Cl} 0.60-1.21 \%, p=0.47)$. The investigators found low heterogeneity between studies $\left(\mathrm{I}^{2}=0,95 \% \mathrm{Cl} 0\right.$ $56.3 \%, p=0.85)$. The overall prevalence of birth defects was 2.9\% (95\% Cl $2.1-4.0 \%)$, range 0 - 22.6\% (95\% $\mathrm{Cl} 9.6$ - 41\%). This is similar to the ranges observed in the general population: $2.7 \%, 2.5 \%$ and $2.5-8 \%$ in the USA, France and South Africa, respectively.

There was 1 infant out of 1301 with a neural tube defect (myelomeningocele), giving a prevalence of $0.08 \%(95 \% \mathrm{Cl} 0.02-0.43 \%)$. This is also similar to the ranges in the general population, but, as the investigators noted, the upper $\mathrm{Cl}$ would give a higher prevalence, including than that of South Africa. The relative risk between those exposed in the first trimester versus second/third trimester did not differ $(\mathrm{RR}=0.91,95 \% \mathrm{Cl} 0.46-1.79 \%, p=0.79)$.

Stillbirth, spontaneous abortion and preterm delivery were also within the range of the general population.
Rates of termination of pregnancy ranged from 5.3\% (95\% Cl 0.64 - 17.7\%) to 33.7\% (95\% Cl 23.7 - 44.9\%). The investigators noted that one study in Soweto found a relative risk of termination 5.73 times higher (95\% $\mathrm{Cl} 1.45-22.75 \%, p=0.0017)$ among women receiving efavirenz compared with other antiretroviral drugs, highlighting a need for careful counselling of women and attitudes among providers.

\section{DATA FROM FRERE HOSPITAL}

After the APR, the second largest data set included in the meta-analysis was from a large regional cohort in South Africa. ${ }^{15}$ This is also the largest study to date of efavirenz-based ART exposure from the second trimester onwards.

In this study Bera et al. evaluated data from the Efavirenz in Pregnancy Registry, which is prospective and based at the Frere Hospital in East London (a referral hospital for a large area of the Eastern Cape) and set up in January 2006. Women who conceived on efavirenz and presented in the first trimester were offered the choice of termination of pregnancy (to 20 weeks' gestation) or switched to another drug. Women who presented at 14 weeks or later and were eligible for ART were initiated on an efavirenz-based regimen. Between 1 January 2006 and 31 December 2008, 744 women were initiated on efavirenz-based regimens from the second trimester onward. Of these, 89 women were still pregnant at the time of evaluation and 32 were lost to follow up.

During the same period, 220 women conceived while receiving efavirenz-based ART and 42 while receiving nevirapine-based ART. Of this group, 17 and 7 women were still pregnant and 8 and 2 women were lost to follow-up receiving efavirenz and nevirapine, respectively.

Women who had received efavirenz-based ART throughout the entire first trimester were classified as 'complete first-trimester exposure' and those who substituted efavirenz for another drug as 'partial firsttrimester exposure'.

This analysis evaluated data from 851 women with pregnancy outcomes.

Of 623 women initiated on efavirenz in pregnancy, birth defects occurred in 16 live births, a prevalence of $2.6 \%$ (95\% Cl 1.5 - 4.2). In 195 women who conceived while receiving efavirenz, birth defects occurred in 5/184 live births and $1 / 4$ stillbirths, a prevalence of 3.3\% $(95 \% \mathrm{Cl}$ 1.2 - 7.0). In this group, 93\% received efavirenz-based ART for longer than 1 month before conception and all pregnancies were unintended. 
There were no significant differences in the prevalence of birth defects between the first- and second/thirdtrimester exposure (prevalence ratio $1.27,95 \% \mathrm{Cl}$ $0.5-3.20, p=0.301)$. Neither were there differences between complete $(4 / 131 ; 3.1 \%)$ and partial $(2 / 53$; $3.8 \%$ ) efavirenz exposure (prevalence ratio 0.81, 95\% Cl $0.15-4.29, p=0.556$ ).

Worth noting is that there was also a birth defect in 1 out of 33 live nevirapine-exposed infants, a prevalence of $3.0 \%(95 \% \mathrm{Cl} 0.1-15.8)$. The prevalence ratio of birth defects following conception on efavirenz compared with nevirapine was 1.08 ( $95 \% \mathrm{Cl} 0.13-8.65, p=0.69)$. However, the numbers of nevirapine exposures are far too small to draw any conclusions.

\section{CONFLICTING FINDINGS IN RETROSPECTIVE STUDY PACTG 219/219C}

Finally, a study of children enrolled in PACTG protocols 219 and $219 \mathrm{C}$ - a multisite US cohort of children born to HIV-positive women set up to study the long term effects of in utero antiretroviral exposure - recently reported a higher prevalence of birth defects than found in other paediatric cohorts. ${ }^{16}$

This observation was difficult to interpret as exposed children were enrolled retrospectively up to 1 year of age (only prospective pregnancies are enrolled in the APR), and for reasons discussed below.

Protocol 219 followed HIV-infected and uninfected children from May 1993 to August 2000. Children were eligible if their mothers were enrolled in a PACTG trial in pregnancy. In September 2000, protocol 219C was introduced, amending 219 to remove the eligibility criterion mandating enrolment in another PACTG trial. Birth defect data were recorded at study visits. Protocol 219 did not include a direct question about birth defects; $219 \mathrm{C}$ included this question.

The primary determinant was first-trimester exposure. Overall antiretroviral exposure, classes of antiretrovirals and specific antiretrovirals to which at least one child with a birth defect had first-trimester exposure were evaluated.

The reference group was children unexposed to the particular antiretroviral (or class of drug) during the first trimester, which included antiretroviralunexposed children, those only exposed in labour, those unexposed to the particular drug but exposed to other antiretrovirals, and children only exposed beyond the first trimester.

Clinicians were blinded to antiretroviral exposure and the outcome was presence of a birth defect within the first year of life.
A total of 117 children with at least one defect were reported out of the study population of 2202 children. This gave an overall defect prevalence of 5.3\% (95\% Cl $4.4-6.3)$ and $4.7 \%(95 \% \mathrm{Cl} 3.8-5.6)$ if just the 103 cases of major defects were included. The prevalence was $4.8 \%(95 \% \mathrm{Cl} 3.7-6.1)$ in children unexposed in the first trimester and $5.8 \%(95 \% \mathrm{Cl} 4.2-7.8)$ in exposed children. The majority of defects occurred in the heart and musculoskeletal system. A higher defect rate $(5 / 32,15.6 \%)$ was reported among children exposed to efavirenz in the first trimester compared with unexposed children, adjusted odds ratio 4.31 (95\% $\mathrm{Cl}, 1.56$ - 11.86). The defects included 1 laryngomalacia, 1 meningomyelocele, 1 hypospadias, 1 club foot, 1 hypertonicity of extremities and 1 cleft palate.

There was also an association in children exposed to lopinavir/ritonavir, but when adjusted for first-trimester folate antagonist exposure, year of birth and perinatal study participation this did not persist $(p=0.07)$, whereas the association with efavirenz continued.

It is difficult to know whether this study advances or confuses the field. Although based on 2202 children, only a third (763) were exposed to any antiretroviral during the first trimester, compared with over 5000 in the APR. Consequently, with the exceptions of zidovudine, lamivudine and nelfinavir, few children were exposed to individual drugs, which accounts for the wide range of odds ratios and Cls. A similar phenomenon has been observed over the years in the APR with new antiretrovirals, which is followed by a gradual movement towards the mean as the denominator increases, suggesting that initial case notification may drive initial reports to the registry.

The findings also generally differ from the APR, in which only didanosine $(4.5 \%, 95 \% \mathrm{Cl} 2.6-7.1 \%)$ has attracted attention owing to a small but persistent increase in risk of birth defects, while Pls in general, and lopinavir/ritonavir $(1.7 \%, 95 \% \mathrm{Cl} 0.8-3.1 \%)$ in particular, have generally been found to be associated with no increase in risk.

As might be anticipated, folate antagonist exposure during the first trimester was associated with an increased prevalence of birth defects, although data were largely incomplete and the observation did not reach statistical significance. The observed confounding of this risk with lopinavir/ritonavir exposure is a reminder not to forget the obvious.

\section{DISCUSSION}

There is to date no evidence of an increase in the incidence of birth defects among infants exposed to efavirenz beyond the first trimester, and excluding the retrospective analysis, also no increase among infants 
born to women receiving efavirenz in the first trimester. However, the authors of the meta-analysis note several limitations to the evidence base, including few studies reporting risk of bias or attempting to control for potential confounders, and most importantly the limited sample size.

They suggest that although these data should provide reassurance to providers regarding first-trimester exposure, the low incidence of neural tube defects in the general population means a larger sample size is still needed to rule out the increased risk of this specific defect.

They write: 'The balance of risks and benefits of efavirenz in pregnancy merits some recalibration, particularly in resource-limited settings where drug formularies are limited, women of child bearing age represent the majority of those infected with HIV, coinfection with tuberculosis is frequent, and the risk of mortality for those who are eligible for ART is high.'

The approach in the new South African guidelines to use of efavirenz is far more cautious than that of the WHO. Whereas the WHO interpreted the risk as being limited to the first trimester, and allowed for the possibility that some risk may still exist only from first-trimester exposure in spite of the low-quality, conflicting evidence, the South African guidelines do not recommend its inclusion at all in pregnancy.

The South African guidelines rely on the 2004 approved package insert for Stocrin, which the Medicines Control Council (MCC) believes indicates an absolute bar on the use of efavirenz throughout pregnancy. However the MCC is open to revisiting this package insert should the applicant - in this case MSD - apply for an amendment and supply sufficient scientific data to justify the amendment.

Although it may be prudent to guide prescribers to avoid first-trimester exposure, there seems to be strong rationale to recommend the use of efavirenz in pregnancy in South Africa beyond this period, for the following reasons:

Simplification to maximise adherence. Efavirenz must be taken once a day whereas nevirapine is usually dosed twice a day with a 2-week induction phase. The other components of ART are dosed daily; use of nevirapine over efavirenz converts a once-daily to a twice-daily regimen.

Consistency with adult treatment regimens. Efavirenz plus tenofovir plus lamivudine or emtricitabine is recommended for almost all non-pregnant adults.
Simplicity of monitoring. Efavirenz does not usually require additional blood tests to the overall regimen. Nevirapine requires baseline alanine aminotransferase $(A L T)$, and liver function tests (LFT) if the baseline ALT is abnormal or if patients develop a rash, any significant mucocutaneous reactions, fever, jaundice or abdominal pain.

\section{Reduction in incidence of toxicity:}

- Efavirenz compared with nevirapine is a safer drug with respect to adverse events, particularly for severe adverse events.

- In a representative South African adult population, 7.6\% of patients who started ART with nevirapine had to stop the drug due to toxicity by 3 years, compared with $1.9 \%$ of those starting ART with efavirenz having to stop efavirenz due to toxicity. Most of the nevirapine substitutions were in the first 3 months of treatment. ${ }^{17}$

- Nevirapine has been associated with an increased risk of toxicity in women with a CD4 count greater than 250 cells/ $\mu \mathrm{l}$ in early studies, and a box warning to this effect has been included. The WHO reviewed the literature and felt reassured to initiate nevirapine in women with high CD4 cell counts. Subsequent to this review an article representing over 10000 patients treated with nevirapine-based ART suggested a high CD4 cell count in treatment-naïve patients to be a risk factor for nevirapine toxicity. ${ }^{18,19}$

\section{Co-tuberculosis (TB) treatment:}

- Efavirenz has better blood levels when used with anti-TB treatment, specifically rifampicin, compared with nevirapine, resulting in slightly lower treatment failure. ${ }^{20}$

- The first-line ART choice in the current South African guidelines for a patient with TB would be tenofovir plus lamivudine or emtricitabine plus efavirenz in all patients whether pregnant or not, at odds with the rationale for not recommending efavirenz in pregnant women who do not have tuberculosis.

- Rash that occurs during co-administration may be due to the TB drugs or nevirapine, and it may occasionally be difficult to determine which to stop.

Availability of fixed-dose combinations (FDCs):

- Efavirenz is included in a couple of FDCs that are pending registration in South Africa - these are tenofovir plus lamivudine plus efavirenz, and tenofovir plus emtricitabine plus efavirenz. The FDA has already tentatively approved such products (tentative only in that patent protection currently prevents them from being marketed in the USA).

- FDCs facilitate adherence and simplify the taking of treatment. They take up less space, with transport and storage savings. 
It is instructive that in the USA, in 2009, among pregnant women receiving NNRTI-based ART, over four times as many receive efavirenz compared with nevirapine. ${ }^{21}$ In a UK cohort, nearly $20 \%$ of women receiving an NNRTI regimen before conception received efavirenz. ${ }^{22}$

Bera et al. above ${ }^{15}$ note that all the pregnancies in the women conceiving on efavirenz in their cohort were unintended, and an investigation into the correct application of guidelines in Johannesburg revealed that the majority (77 - 90\%) of women were incorrectly assigned to efavirenz with respect to contraception use, and 39\% were either trying to conceive or planned to do so in the next year. ${ }^{23}$

\section{CONCLUSION}

In short, the recommendation for nevirapine in pregnancy, based on poor data concerning the first trimester and without rationale beyond the first trimester, will result in women being exposed to a more toxic and more complicated regimen, which has significant additional operational challenges and differs from the otherwise very uniform approach to first-line ART provision to adults in the national programme. If indeed there is a concern about neural tube defects, the appropriate guideline response should be to focus on the avoidance of conception in women on efavirenz in line with the WHO recommendations and the prescriber information that accompanies efavirenz when distributed as Stocrin.

This real risk to maternal health and in turn child health must be weighed up against the theoretical risk of fetal toxicity. We believe that the decision to uniformly use nevirapine in pregnancy is a poor one in the absence of better data, and should be reviewed by the MCC and the Department of Health.

Thanks to Graham Taylor and Karen Beckerman.

\section{REFERENCES}

1. National Department of Health, South Africa: South African National AIDS Council. Clinical Guidelines: PMTCT (Prevention of Mother-to-Child-Transmission) 2010. http://www.doh.gov.za/docs/index.html (accessed 23 October 2010).
2. US Department of Health and Human Services. Food and drug Administration. Important Information About Sustiva (efavirenz) and Pregnancy. March 2005. http://www.fda.gov/ForConsumers/ByAudience/ForPatientAdvocates/ HIVandAIDSActivities/ucm 124885.htm (accessed 23 October 2010).

3. The Antiretroviral Pregnancy Registry. http://www.apregistry.com/ (accessed 23 October 2010).

4. Ford N, Mofenson L, Kranzer K, et al. Safety of efavirenz in first trimester of pregnancy: a systematic review and meta-analysis of outcomes from observational cohorts. AIDS 2010; 24(10): 1461-1470.

5. World Health Organization. Antiretroviral Drugs for Treating Pregnant Women and Preventing HIV Infections in Infants. 2010 version. http://whqlibdoc.who.int/ publications/2010/9789241599818_eng.pdf (accessed 23 October 2010).

6. World Health Organization. Antiretroviral Therapy for HIV Infection in Adults and Adolescents. 2010 revision. http://whqlibdoc.who.int/ publications/2010/9789241599764_eng.pdf (accessed 23 October 2010).

7. Recommendations for Use of Antiretroviral Drugs in pregnant HIV-1 Infected Women for Maternal Health and Interventions to Reduce Perinatal HIV Transmission in the United States. May 24, 2010. http://www.aidsinfo.nih.gov/ Guidelines/GuidelineDetail.aspx?GuidelineID=9 (accessed 23 October 2010).

8. Ministry of Health Zambia. National Protocol Guidelines. Integrated Prevention of Mother-to-Child-Transmission of HIV/AIDS. September 2009. Ministry+of+ Health+Zambia +National+Protocol+Guidelines +Integrated+Prevention+of+Mo ther-to-Child-Transmission+of+HIV\%2FAIDS.++September+2009 (accessed 23 October 2010).

9. European AIDS Clinical Society. Clinical Management and Treatment of HIV Infected Adults in Europe. Version 5.2. http://www.europeanaidsclinicalsociety. org/guidelinespdf/1_Treatment_of_HIV_Infected_Adults.pdf (accessed 23 October 2010).

10. British HIV Association and Children's HIV Association guidelines for the management of HIV infection in pregnant women. HIV Medicine (2008). http:// www.bhiva.org/PregnantWomen2008.aspx (accessed 23 October 2010).

11. Bristol-Meyers Squibb. Sustiva - package insert.

12. Merck Sharp Dohme. Stocrin - package insert.

13. Aspen Efavirenz. Package insert.

14. Ford N, Mofenson L, Kranzer K et al. Safety of efavirenz in first-trimester of pregnancy: a systematic review and meta-analysis of outcomes from observational cohorts. 18th International AIDS Conference. 18-23 July 2010, Vienna. Oral abstract WEAX0102.

15. Bera $E$, McCausland $K$, Nonkwelo $R$, et al. Birth defects following exposure to efavirenz-based antiretroviral therapy during pregnancy: a study at a regional South African hospital. AIDS 2010; 24(2): 283-289.

16. Brogly SB, Abzug MJ, Watts $\mathrm{DH}$ et al. Birth defects among children born to human immunodeficiency virus-infected women. Pediatric AIDS Clinical Trials Protocols 219 and 219C. Pediatr Infect Dis J 2010; 29(8):721-7.

17. Boulle A Orrell $C$, Kaplan $R$, et al. Substitution due to ART toxicity or contraindication in the first three years of ART in large South African cohort. Antiviral Ther 2007; 12: 753-760.

18. Boehringer Ingelheim. Nevirapine - package insert.

19. Kesselring AM, Wit FW, Sabin CA, et al. Risk factors for treatment limiting toxicities in patients starting nevirapine-containing antiretroviral therapy. AIDS 2009; 23(13): 1689-1699.

20. Boulle A, Van Cutsem $G$, Cohen $K$, et al. Outcomes of nevirapine and efavirenzbased antiretroviral therapy when coadministered with rifampicin-based antitubercular therapy. JAMA 2008; 300: 530-539.

21. Griner $R$, Williams $P$, Read J, et al. Prenatal exposure to antiretrovirals among HIV-exposed but uninfected children: Surveillance Monitoring for ART Toxicities Study. 17th Conference on Retroviruses and Opportunistic Infections, 16-19 February 2010, San Francisco. Poster abstract 921.

22. Sabin $C$, Huntington $S$ Bansi $L$, et al. Use of antiretroviral therapy during and after pregnancy among HIV-infected women already aware of their infection before conceiving. 2nd Joint Conference of BHIVA with BASHH, 20-23 April 2010 Manchester. Poster abstract p154.

23. Hanrahan $\mathrm{C}$, Schwartz $\mathrm{S}$, Rees $\mathrm{H}$, et al. South African antiretroviral treatment guidelines for women: how well are they being followed and for whom? 18th International AIDS Conference, 18-23 July 2010, Vienna. Poster abstract THPE0120.

(1)

